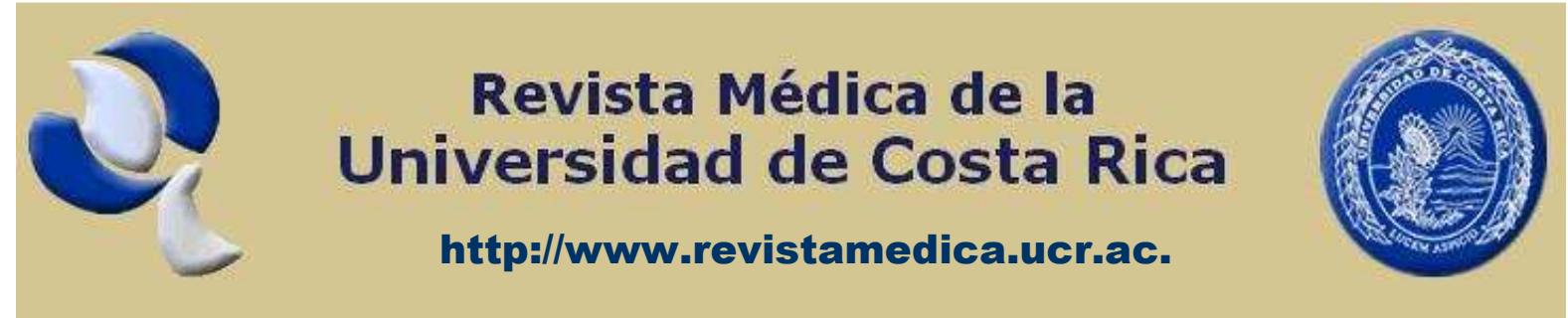

Educación médica continua

\title{
Abordaje y manejo inicial de la angina inestable $y$ el infarto del miocardio sin elevación del segmento ST.
}

\author{
Salas Segura, Donato A.
}

Especialista en Medicina Crítica y Cuidado Intensivo del Hospital Rafael Ángel Calderón Guardia, San José, Costa Rica.

Profesor de la Escuela de Tecnologías Médicas y del Departamento de Farmacología y Toxicología Clínica de la Escuela de Medicina de la Universidad de Costa Rica, San Pedro, San José, Costa Rica.

Resumen: Los síndromes coronarios agudos sin elevación del segmento S-T incluyen a la angina inestable y al infarto sin elevación del S-T. El diagnóstico temprano y la estratificación del riesgo son fundamentales en su manejo. Este artículo revisa el manejo actual de estos síndromes de acuerdo a las guías y estrategias ya establecidas.

Palabras clave: Infarto del miocardio, angina inestable, síndrome coronario agudo

Abstract: The non-ST segment elevation acute coronary syndromes include unstable angina pectoris and the non-ST segment elevation myocardial infarction, acute diagnosis and risk stratification are very important for the treatment. This review will cover guidelines and strategies for risk assessment, contemporary approaches to acute patient management.

Keywords: Myocardial infarction, unstable angina, acute coronary syndromes

Recibido: Febrero 2009. Aceptado: Febrero 2009. Publicado: Marzo 2009.

Revista electrónica publicada por el Departamento de Farmacología de la Escuela de 


\section{Introducción}

La cardiopatía isquémica y la enfermedad cerebro-vascular son la principal de causa de muerte no traumática en los países desarrollados y en la mayoría de los países en vías de desarrollo. En el año 2003, fallecieron aproximadamente 17 millones de personas por enfermedad cardiovascular, una tercera parte de todas las muertes en el mundo [1].

Con un fin práctico, la cardiopatía isquémica en su presentación aguda, ha sido clasificada de acuerdo a los cambios electrocardiográficos y de elevación de troponina, en 3 entidades de gravedad y letalidad ascendentes: angina inestable (Al), infarto del

\section{Estableciendo la severidad.}

El cuadro clínico y el diagnóstico del síndrome coronario agudo, aunque de conspicua importancia, escapan a los objetivos de este trabajo de modo que hará énfasis en el manejo de la enfermedad una vez hecho el diagnóstico adecuado.

La terapia anti-isquémica de los SCA sin elevación del ST tiene dos aspectos fundamentales. El primero es la estratificación del riesgo y el segundo, la realización de angioplastia coronaria temprana en aquellos grupos considerados de mediano o alto riesgo.

Existen varias escalas utilizadas para estratificar el riesgo en el paciente isquémico, de ellas la más utilizada y plenamente validada, es la Escala de riesgo TIMI para AI /NSTEMI (ver cuadro 1).

Otros dos modelos de puntaje de riesgo, basados ambos también en extensos estudios clínicos, que han recibido el beneplácito de su uso por la Sociedad miocardio sin elevación del segmento ST (NSTEMI por sus siglas en inglés) e infarto del miocardio con elevación del ST. Estas tres entidades constituyen el "Síndrome Coronario Agudo" (SCA).

Mientras que el manejo clínico de la $\mathrm{Al}$ como el NSTEMI es determinado por el grado de riesgo, el infarto con elevación del ST requiere de la revascularización más urgente posible ya sea por medio del uso de fibrinolíticos o de angioplastia coronaria.

El presente artículo hará un revisión sucinta pero lo más completa posible del manejo actual de los 2 primeros componentes del SCA, la angina inestable y el NSTEMI.

Americana del Corazón [4], son el GRACE y el PURSUIT.

Todos estos modelos de riesgo son útiles para decidir: Dónde internar al paciente, escoger los fármacos antiplaquetarios más apropiados y determinar la necesidad o no de de estrategias intervencionistas tempranas $y$, al momento del egreso, informar al paciente cual es su pronóstico a mediano y largo plazo $[5,6]$.

Una vez estratificado el riesgo, el abordaje terapéutico dependerá del puntaje obtenido.

Antes de revisar el manejo de los pacientes de acuerdo al su puntaje TIMI es conveniente revisar las diferentes medidas terapéuticas aplicables a los SCA y luego establecer su uso según el riesgo.

Revista electrónica publicada por el Departamento de Farmacología de la Escuela de 


\section{Cuadro No. 1 Escala (score) de riesgo TIMI para AI/NSTEMI.}

La escala de TIMI fue desarrollada durante el estudio clínico TIMI 11B (2) y quedó validada en por el estudio ESSENCE (3). Está compuesta por sietes ítems con valor asignado de 1 punto por cada uno de ellos:

1.- Edad > 65 años

2.-Al menos tres de los siguientes factores de riesgo coronario:

Hipertensión Arterial

Hipocolesterolemia

Historia familiar positiva

Diabetes mellitus

Tabaquismo actual

3.- Estenosis coronaria significativa ( $>50 \%)$ establecida ya sea por cualquiera de los siguientes:

Coronariografía previa

Angioplastía coronaria previa

Infarto agudo del miocardio previo

Cirugía coronaria previa

4.- Desviación del segmento ST (>0.5 mm)

5.-Síntomas severos:

Dos o más episodios en reposo en las últimas 24 horas

6.- Uso de aspirina en los últimos siete días

7.- Marcadores séricos elevados (básicamente troponina)

Un puntaje de 0 a 2 se considera de bajo riesgo, un puntaje de 3 a 4 de riesgo intermedio y un puntaje de 5 a 7 de riesgo alto.

Cuadro No. 2

Puntaje del TIMI score y riesgo de mortalidad, nuevo infarto o isquemia recurrente severa que requiera revascularización en los primeros 14 días y mortalidad al año luego de la asignación del puntaje [4].

\begin{tabular}{|l|ccc|}
\hline $\begin{array}{c}\text { Puntaje } \\
\text { TIMI }\end{array}$ & $\begin{array}{c}\text { Frecuencia de } \\
\text { eventos }\end{array}$ & $\begin{array}{c}\text { Mortalidad } \\
\text { 1er año }\end{array}$ & Riesgo \\
\cline { 1 - 2 } $\mathbf{0 - 1}$ & $\mathbf{4 . 7}$ & $\mathbf{3 , 9 \%}$ & Bajo \\
\cline { 1 - 2 } $\mathbf{2}$ & $\mathbf{8 , 3}$ & & \\
\hline $\mathbf{3}$ & $\mathbf{1 3 , 2}$ & $\mathbf{6 , 5 \%}$ & Intermedio \\
\hline $\mathbf{4}$ & $\mathbf{1 9 , 9}$ & & \\
\hline $\mathbf{5}$ & $\mathbf{2 6 , 2}$ & $\mathbf{2 1 \%}$ & Alto \\
\hline $\mathbf{6 - 7}$ & $\mathbf{4 0 , 9}$ & & \\
\hline
\end{tabular}

Revista electrónica publicada por el Departamento de Farmacología de la Escuela de 


\section{Medidas generales.}

El abordaje inicial de cualquier cuadro sugestivo de isquemia miocárdica tiene una serie de elementos comunes cuyo fin básico es aliviar el dolor y limitar el daño al músculo.

En forma clásica y casi legendaria, se ha usado un nemotecnia para recordar 4 medidas terapéuticas fundamentales en el abordaje de los pacientes con cardiopatía isquémica: MONA, donde la $M$ corresponde a morfina endovenosa para el dolor, la $\mathrm{O}$ es para oxígeno, la $\mathrm{N}$ para nitroglicerina y la A para aspirina. Este sencillo acrónimo es útil y válido especialmente en aquellos centros de salud con recursos muy limitados pero, a la luz de nuestros conocimientos actuales, es también incompleto e insuficiente. (Ver cuadro No. 3). La mayor parte de las medidas farmacológicas entre más temprano se inicien, más útiles resultarán.

\section{Cuadro No 3. Terapia analgésica y anti-isquémica temprana en los SCA.}

1. Administrar oxígeno para mantener una saturación de arterial >92\% o si hay insuficiencia respiratoria.

2. Aspirina, al arribo o desde antes, y se continuará indefinidamente. La dosis inicial es 165 $-325 \mathrm{mg}$.

3. En pacientes que no puedan usar aspirina, se utilizará clopidogrel con una dosis de carga de $300 \mathrm{mg}$ y de $75 \mathrm{mg}$ diarios.

4. Usar nitroglicerina endovenosa si hay isquemia persistente, insuficiencia cardiaca o hipertensión arterial. Si solo se dispone de nitroglicerina oral dar 0,4 mg c/ 5 minutos, máx. 3 dosis.

5. Dar un beta-bloqueador oral si no hay contraindicaciones (p.e, shock, insuficiencia cardiaca, o frecuencia $<60$ latidos/min).

6. Si no es posible usar un beta-bloqueador, a veces es posible utilizar un calcio-antagonista no-dihidropiridínico tipo verapamil o diltiazem.

7. Dar un inhibidor de enzima convertasa (IECA) dentro de las primeras 24 horas del evento especialmente si hay insuficiencia cardiaca, o una fracción de eyección $<40 \%$. No dar en pacientes hipotensos. Si no tolera el IECA, dar un inhibidor de receptor de aldosterona (ARA).

8. Se deberá dar protección gástrica si hay intolerancia a la aspirina o al clopidrogrel.

\section{Terapia antitrombótica.}

En pacientes con SCA, la aspirina será utilizada temprana e indefinidamente, sola o como parte de la triple terapia antiplaquetaria junto con el clopidogrel y los Inhibidores de receptores de glicoproteínas Ilb/llla (Gp IIlb/llla).

La dosis inicial (en agudo) de aspirina es de 160 a 325 mgs y la dosis de mantenimiento (diaria) recomendada

Revista electrónica publicada por el Departamento de Farmacología de la Escuela de 
varía de 75 a $160 \mathrm{mg}$. La eficacia y validez de estos rangos de dosis está fuertemente establecido desde hace 20 años [4]. Dosis mayores simplemente se asocian a mayores efectos secundarios. El clopidogrel debería ser recibido por todos los pacientes al menos por 7 días. En aquellos pacientes que sean llevados a angioplastia coronaria se recomienda un dosis de carga de 300 a $600 \mathrm{mg}$ seguida por una dosis diaria de $75 \mathrm{mg}$ por al menos un mes si no se le coloca una endoprótesis vascular (stent) o ésta es no-recubierta o por un año si se le colocan uno o varios "stents" recubiertos [7].

Los inhibidores de Gp Illb/llla actúan bloqueando la ruta final de la agregación plaquetaria y solo existen en presentación endovenosa por lo que su uso se limita a unas cuantas horas. Están indicados para los pacientes con AI/INSTEMI de bajo, mediano y alto riesgo o con troponina elevada. En los pacientes de bajo riesgo se pueden utilizar en vez del clopidogrel pero no es necesario utilizarlos junto con él. En los casos de mediano y alto riesgo se emplean en conjunto con aspirina, clopidogrel y heparina. Son especialmente útiles en pacientes sometidos a angioplastia coronaria con colocación de stent $[4,8]$. Los tres fármacos que están actualmente en el mercado, abciximab, epifibatide y tirofibán, son útiles después de la angioplastia con ferulización, sin embargo, las recomendaciones actuales sugieren que si no se colocaron "stents", el medicamento de elección es el abciximab.

\section{Anticoagulación.}

El uso de la heparina en los SCA está claramente establecido aunque el uso de heparinas de bajo peso molecular (HBPM) a veces causa confusión.

La heparina no fraccionada actúa fundamentalmente en la interacción trombina y antitrombina produciendo la inhibición de la agregación plaquetaria inducida por la trombina. También tiene un efecto inhibitorio sobre el factor $\mathrm{Xa} y$ en menor grado sobre el factor XI $y$ otros factores de la llamada vía intrínseca.

La heparina no fraccionada endovenosa tiene un rápido inicio de acción, su efecto también desaparece rápidamente (4 horas), su dosis es titulable y puede ser usada en pacientes inestables o que serán sometidos a angioplastia prontamente. Tiene dos problemas fundamentales: puede ocurrir rebote cuando se suspende y dos, tiene un riesgo elevado de producir plaquetopenia (3 a $5 \%$ de los pacientes con más de 5 días de infusión).

La infusión de heparina se prepara diluyendo 25000 unidades en $250 \mathrm{ml}$ de solución salina al $0,9 \%$ con lo cual cada mililitro de infusión equivale a $100 \mathrm{U}$. La dosis recomendada según las guías europeas [8] es iniciar con un bolo de 60 a $70 \mathrm{U} / \mathrm{kg}$ hasta un máximo de 5000 $U$ y continuar con una dosis de entre 12 a $15 \mathrm{U} / \mathrm{kg}$ por hora (para un máximo teórico de $1000 \mathrm{U} / \mathrm{h}$ ). Las guías de norteamericanas son un poco más cautas y sugieren iniciar con un bolo de $60 U / \mathrm{kg}$ (máximo $4000 \mathrm{U}$ ) y utilizar una velocidad de infusión de $12 \mathrm{U} / \mathrm{kg} / \mathrm{h}$ [4]. La meta es lograr un TPT de 2 a 2.5 veces mayor al valor del TPT basal del paciente.

Las heparinas de bajo peso molecular (HBPM) se llaman así porque su peso molecular es de alrededor de un tercio del heparina no fraccionada. Actúan fundamentalmente sobre el factor Xa y

Revista electrónica publicada por el Departamento de Farmacología de la Escuela de 
en mucho menor grado sobre el complejo antitrombina-trombina.

En SCA, las HBPM son tan o más efectivas que la heparina no fraccionada y se asocian a menos complicaciones como sangrado o trombocitopenia [9].Se prefieren a la heparina no fraccionada porque no necesitan monitoreo de TPT, y son fáciles de administrar. No deben ser usadas en pacientes con insuficiencia renal con un aclaramiento endógeno de creatinina
(AEC) menor a $30 \mathrm{ml} /$ minuto $\mathrm{ni}$ en pacientes que serán llevados a angioplastia prontamente pues por su acción prolongada y difícil de revertir, aumentan el riesgo de sangrado.

No hay estudios que comparen las HBPM entre sí para determinar cual de ellas es superior para su uso en SCA. Hasta la fecha, la enoxaparina es la heparina con más estudios a su haber y por ello es la más utilizada y recomendada [10].

\section{Cuadro No. 4 Otros agentes anticoagulantes: Bivalirudina y Fondaparinux.}

En pacientes con SCA de riesgo moderado o severo y preparados para angioplastia temprana, el inhibidor directo trombina Bivalirudina es igual de eficaz que la heparina (fraccionada o no) asociada con Inhibidores GIIb/IIIa y produce menos sangrado. Se debe dar en infusión y en combinación con aspirina y clopidogrel [11].

El Fondaparinux, un inhibidor indirecto de la trombina, es un anticoagulante útil en pacientes de alto riesgo de sangrado y de manejo conservador o no invasivo. Ha demostrado ser superior a las HBPM en AI/NSTEMI (12). Se administra por vía subcutánea una vez al día (2,5 mg). No debe usarse en pacientes con AEC $<30 \mathrm{ml} / \mathrm{min}$. También se emplea en profilaxis de trombosis venosa.

\section{Angioplastía y "stents".}

La angioplastia vascular se realiza provocando la fractura de la placa de ateroesclerosis por medio de un balón inflable, con esto se logra un distribución parcial de la placa a lo largo de la longitud del vaso que asociada a la expansión de la lámina elástica interna, resuelve la obstrucción de la arteria. Las endoprótesis vasculares endovasculares 0 "stents" son estructuras metálicas colocadas dentro de un segmento de una arteria coronaria obstruida con el objetivo de mantener la luz arterial permeable y prevenir así la reoclusión temprana del vaso.

La angioplastia coronaria es la modalidad más utilizada de revascularización coronaria.

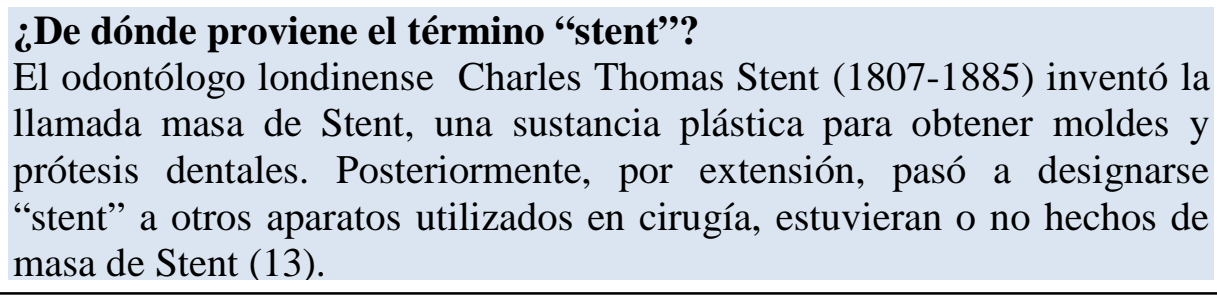

Revista electrónica publicada por el Departamento de Farmacología de la Escuela de Medicina de la Universidad de Costa Rica, 2060 San José, Costa Rica. ${ }^{\circledR}$ All rights reserved. 
En pacientes de alto riesgo y en los de riesgo moderado con algunas condiciones (p.e diabetes o dolor rebelde a tratamiento) la angioplastia coronaria constituye la medida terapéutica más importante en el manejo de los SCA $[4,8,11]$.

A grandes rasgos existen dos tipos de "stents": los no-recubiertos (bare-metal en inglés) y los recubiertos (drug-eluting stents), estos últimos se llaman así porque su estructura metálica esta revestida con fármacos inmunomoduladores cuyo objeto es evitar la hiperplasia de la íntima y prevenir la estenosis tardía del vaso.

Se utilizan dos medicamentos: sirolimus y paclitaxel El sirolimus (rapamicina) inhibe la proliferación de fibroblastos a través de la inhibición de los receptores mTOR (target of rapamycin) y el paclitaxel inhibe la hiperplasia neointimal [14].

A la fecha, el uso de "stents" recubiertos, a pesar de alto precio, no ha demostrado disminuir la tasa de infarto o muerte cuando se le compara contra "stents" no-recubiertos [15].

\section{Integración.}

Todos los pacientes con dolor torácico o alguna otra condición que haga sospechar que se trata de un SCA (p.e, Edema agudo de pulmón o arritmias) deberán recibir todas aquellas medidas generales discutidas previamente y deberá clasificarse de acuerdo a la escala de riesgo TIMI.

Pacientes con un puntaje TIMI 0-2.

Se deben observar en emergencias y eventualmente pueden egresarse 0 internarse a salón para completar los estudios respectivos que incluirán una prueba para la detección de isquemia (prueba de esfuerzo, eco-estrés o MIBI cardiaco). Pueden recibir clopidogrel pero no necesitan anticoagulación ni angiografía temprana [16].

\section{Pacientes con un puntaje TIMI 3-4.}

Son paciente de riego intermedio el cual puede estar matizado por la presencia de otros factores que al final determinen la necesidad de un angiografía y angioplastia coronarias (ver Cuadro No.5).

Todos estos pacientes se deben internar y recibir anticoagulación con heparina. La escogencia del tipo de heparina dependerá de la necesidad y el momento de realización de la angiografía coronaria, si ésta se va a realizar en forma precoz ha de utilizarse heparina no fraccionada.

Además de la aspirina y el clopidogrel, el paciente con troponina positiva o un puntaje de 4, debería recibir inhibidores de Gp Illb/lla, los cuales se pueden iniciar al menos 4 horas antes del procedimiento angiográfico y continuar por un espacio de 24 a 48 horas [17, 18, 19].

\section{Pacientes con un puntaje TIMI 5-7}

Todos estos pacientes son tributarios de angiografía coronaria temprana (24 a 48 horas) para determinar si requieren de endoprótesis o de cirugía de derivación coronaria $[4,20,21]$.

Su manejo incluye todas las medidas discutidas hasta ahora por lo cual es innecesario repetirlas de nuevo aquí.

Revista electrónica publicada por el Departamento de Farmacología de la Escuela de 


\section{Cuadro No. 5. Condiciones que elevan el riesgo en paciente con un puntaje TIMI 3-4 [4].}

1. Angina o isquemia recurrentes a pesar de terapia médica intensa y adecuada.

2. Troponina elevada ( $\mathrm{T}$ o I).

3. Depresión del ST nueva.

4. Inestabilidad hemodinámica.

5. Taquicardia ventricular sostenida.

6. Angioplastía previa en los últimos 6 meses.

7. Cirugía de derivación coronaria ( By-pass) previa.

8. Signos o síntomas de insuficiencia cardiaca o un fracción de eyección por ecocardiografía $<40 \%$.

9. Hallazgos de alto riesgo por estudios no invasivos.

\section{Referencias}

1- Mackay J, Mensah G. The atlas of heart disease and stroke. Geneva: World Health Organization, 2004.

2- Antman E, McCabe C, Gurfinkele E, Turpe A, Bernink PJ, Salein D, et al. Enoxaparin Prevents Death and Cardiac Ischemic Events in Unstable Angina/Non-Q-Wave Myocardial Infarction.Results of the Thrombolysis In Myocardial Infarction (TIMI) 11B Trial. Circulation. 1999;100:1593-1601.

3- Fox KA. Low molecular weight heparin (enoxaparin) in the management of unstable angina: the ESSENCE study. Heart. 1999; 82 (Suppl 1):I12-I14

4- ACC/AHA 2007 Guidelines for the Management of Patients with Unstable Angina/Non-ST-Elevation Myocardical Infarction. J Am Coll Cardiol. 2007; 50(7): e1-e157.

5- Araújo-Gonçalves Pedro, Ferrerira J, Aguiar C, Seabra-Gómes. TIMI, PURSUIT; and GRACE risk scores: sustained prognostic value and interaction with revascularization in NSTE-ACS. Eur Heart J 2005; 26: 865-872.

6- García-Almagro FJ, Gimeno JR, Villegas M, Muñoz L, Sánchez E, Teruel F, et al. Aplicación de una puntuación de riesgo coronario (TIMI Risk Score) en una población no seleccionada de pacientes que consultan por dolor torácico en un servicio de urgencias. Rev Esp Cardiol 2005; 58 (7): 775-81.

7- Smith SC et al. AHA/ACC Guidelines for secundary prevention for patients with coronary and other atherosclerotic vascular diseases: 2006 update, endorsed by the National Heart, Lung and Blood Institute. Circulation 2006; 113: 2363-2372.

8- Bassand JP et al. Guidelines for the diagnosis and treatment of non-ST elevation acute coronary syndromes. The Task Force for the Diagnosis and Treatment of non-ST Elevation Acute Coronary Syndromes of the European Society of Cardiology. Eur Heart J 2007; 28: 1598-1660.

9. Murphy SA, Gibson CM, Morrow DA, Van de Werf F, Menown IB, Goodman SG, Mahaffey $\mathrm{KW}$. Efficacy and safety of the low-molecular weight heparin enoxaparin compared with unfractionated heparin across the acute coronary syndrome spectrum: a meta-analysis. Eur Heart J 2007; 28: 2077-2086.

10. Eikelboom JW, Quinlan DJ, Mehta SR, Turpie AG, Menown IB, Yusuf S. Unfractionated and low-molecular weight heparin as adjuncts to thrombolysis in aspirin-treated patients with STelevation acute myocardical infarction: a meta-

Revista electrónica publicada por el Departamento de Farmacología de la Escuela de 
analysis of the randomized trials. Circulation 2005; 112: 3855-3867.

11. Alpert JS, Pollack CV. Antithrombotic therapy in ACS. New York USA: The Exeter Group, 2008.

12. Yusuf S, Mehta SR, Chrolavicius S, Afzal R, Pogue J, Granger CB. Comparison of fondaparinux and enoxaparin in acute coronary syndromes. N Eng J Med 2006; 354(14):14641476.

13. Navarro FA. Diccionario crítico de dudas inglés - español de medicina. Madrid, España: McGraw-Hill Interamericana, 2000.

14-Opie LH, Gersh BJ. Drugs for the heart. Philadelphia USA: Saunders-Elsevier, 2009.

15-Kastrati A, Mehill J, Pache J, Kaiser C, Valgimigli M, Kelboek $\mathrm{H}$, et al. Analysis of 14 trials comparing sirolimus-eluting stents with bare-metal stents. N Eng J Med 2007; 356:10301039.

16-Boden WE, O'Rourke RA, Crawford MH, Blaustein A, Deedwana PV, Zoble R, et al. Outcomes in patients with acute non-Q-wave myocardial infarction randomly assigned to an invasive as compared with a conservative management strategy. Veterans Affairs Non-QWave Infarction Strategies in Hospital (VANQWISH) Trial Investigators. N Engl J Med 1998; 338:1785-1792.
17- Martínez-Rumayor A, Januzzi JL. Non-ST Segment Elevation Acute Coronary Syndromes: A Comprehensive Review. South Med J 2006; 99:1103-1110.

18- Januzzi JL, Chae CU, Sabatine MS. Elevation in serum troponin I predicts the benefit of tirofiban. J Thromb Thrombolysis 2001; $11: 211-215$.

19- Morrow DA, Cannon CP, Rifai N, et al. TACTICS-TIMI 18 Investigators. Ability of minor elevations of troponins I and $T$ to predict benefit from an early invasive strategy in patients with unstable angina and non-ST elevation myocardial infarction: results from a randomized trial. JAMA 2001; 286:2405-12.

20- de Winter RJ, Windhausen F, Cornel JH, Dunselman PHJ, Janus C, Bendermarcher PEP, et al. Invasive versus Conservative Treatment in Unstable Coronary Syndromes (ICTUS)Investigators. Early invasive versus selectively invasive management for acute coronary syndromes. N Engl J Med 2005; 353:1095-1104.

21-Chadow HL, Hauptman RE, VanAuker M, Rafii SE, Gunsburg MY, Giarraffa L, Strom JAl. Drip and ship: a new strategy for the treatment of acute coronary syndromes. J Thromb Thrombolysis

2000; 10:77-82.

\section{Correspondencia:}

Donato A. Salas-Segura.

Apdo. Postal 10706-1000 SJ, Costa Rica

e-mail: dasscom@racsa.co.cr

Revista electrónica publicada por el Departamento de Farmacología de la Escuela de 